\title{
Dot blot para identidad del polisacárido de Streptococcus pneumoniae serotipo 14 y toxoide tetánico en vacunas conjugadas
}

\section{(Dot blot to identify polysaccharides from Streptococcus pneumoniae serotypes 14 and tetanus toxoid on conjugate vaccines)}

\author{
*O. Cabrera ${ }^{1}$, M. Pisonero ${ }^{1}$, M. Rodríguez¹, R. Pérez¹, E. González², J. \\ Pedroso ${ }^{1}$, D. García ${ }^{3}$ y M. Cuello
}

${ }^{1}$ Departamento de Análisis; ${ }^{2}$ Grupo de Anticuerpos Monoclonales; ${ }^{3}$ Dirección de Investigaciones. Instituto Finlay de Vacunas. Ave 27, No. 19805, La Lisa. La Habana, Cuba; ${ }^{4}$ Facultad de Ingenierías y Tecnologías, Universidad Técnica “Luis Vargas Torres” Esmeraldas, Ecuador *ocabrera@finlay.edu.cu

\section{RESUMEN}

Las autoridades regulatorias recomiendan el uso de técnicas inmunoquímicas para determinar la identidad de los antígenos presente en las vacunas conjugadas. Con el surgimiento de las vacunas multivalentes se ha hecho necesario el uso de técnicas inmunoquímicas con el empleo de anticuerpos monoclonales (AcM) para incrementar la sensibilidad de la determinación. El objetivo de este estudio fue establecer las condiciones analíticas que permitan utilizar el Dot Blot como técnica para determinar la identidad del polisacárido capsular de Streptococcus pneumoniae serotipo 14 (PsC 14) y del toxoide tetánico (TT) utilizada como proteína carrier en vacunas conjugadas multivalentes. AcMs contra el PsC 14 y el TT fueron utilizados, además fueron utilizados varios lotes de conjugados monovalentes del PsC 14, lotes de un candidato vacunal heptavalente contra el neumococo con TT como proteína transportadora y lotes de TT, todos producidos en el Instituto Finlay de Vacunas. Los resultados mostraron que para la determinación de la identidad antigénica fueron suficientes $10 \mu \mathrm{L}$ de muestras de los conjugados monovalentes en una dilución de 1/10 (vol/vol) e igual volumen para las vacunas heptavalentes. Quedó demostrado que a la concentración de $2 \mu \mathrm{g} / \mathrm{mL}$ para el AcM contra el PsC 14 y a 2,5 $\mu \mathrm{g} / \mathrm{mL}$ para el AcM contra TT fueron suficientes para la determinación; mientras que los tiempos de incubación fueron ajustados a 37oC por $30 \mathrm{~min}$. Como conclusiones de este trabajo podemos plantear que quedaron establecidas las condiciones de trabajo en el laboratorio para determinar la identidad del polisacárido capsular de S. pneumoniae serotipo 14 y para el toxoide tetánico en las vacunas conjugadas multivalentes por el método del Dot Blot.

Palabras claves: Identidad, dot blot, s. pneumoniae, anticuerpos monoclonales. 


\begin{abstract}
Regulatory authorities recommend the use of immunochemical techniques to determine the identity of the antigens present on the conjugate vaccines. With the emergence of multivalent vaccines, it has become necessary to use immunochemical techniques using monoclonal antibodies to increase the sensitivity in the determination. The aim of this study was to establish the analytical conditions that would allow to use the Dot Blot as a technique to determine the identity of capsular polysaccharides from Streptococcus pneumoniae serotype 14 (PsC 14) and the tetanus toxoid (TT) used as carrier protein in multivalent conjugate vaccines. The MAb against the PsC 14 and TT were used; besides were used some batchs of monovalent conjugates of PsC 14; batchs of a heptavalent conjugate pneumococcal vaccine candidate with TT as carrier protein and batchs of TT, all produced at Finlay Institute of Vaccines. The results showed that for the determination of the antigenic identity were optimal volumes of $10 \mu \mathrm{L}$ of monovalent conjugate samples at a dilution of $1 / 10(\mathrm{vol} / \mathrm{vol})$ and equal volume for heptavalent vaccines. For MAb was demonstrated that $2 \mu \mathrm{g} / \mathrm{mL}$ concentration of MAb against the PSC 14 and 2.5 $\mu \mathrm{g} /$ $\mathrm{mL}$ for MAb against TT were sufficient to successfully perform the determinations; while incubation times were adjusted at $370 \mathrm{C}$ for $30 \mathrm{~min}$. The work allows us to conclude that working conditions in the laboratory were established to determine the identity of the capsular polysaccharides of S. pneumoniae serotype 14 and for tetanus toxoid as carrier protein in the heptavalent conjugate vaccines by Dot Blot method.
\end{abstract}

Keywords: Identity, dot blot, s. pneumoniae, monoclonal antibody. 


\section{INTRODUCCION}

La neumonía es la principal causa de mortalidad infantil en todo el mundo. En el 2015 se reportó 922000 muertes de niños menores de 5 años por neumonía, lo que supone $15 \%$ de todas las defunciones de niños de esta edad en todo el mundo, pero su prevalencia es mayor en el África subsahariana y Asia meridional (OMS, 2015).

El Streptococcus pneumoniae es responsable de enfermedades invasivas como neumonía, meningitis y otitis media, generando a nivel mundial una alta mortalidad (Ortqvist 2005). En Cuba después de la introducción de la vacuna VA-MENGOC-BC ${ }^{\circledR}$ contra los meningococos B y C y Quimi-Hib contra Haemophilus influenzae tipo b; S pneumoniae se ha convertido en el agente causal fundamental de estas patologías (MINSAP, 2015).

Los esfuerzos para combatir estas enfermedades se han concentrado en la obtención de vacunas conjugadas, que han demostrado su eficacia en la protección de estas poblaciones, ya que los polisacáridos capsulares (PsC) son moléculas no inmunogénicas en niños menores de 5 años, que es la población más vulnerable (Cabrera, 2007). Existen actualmente más de 90 serotipos de S. pneumoniae. Asimismo, todas las vacunas desarrolladas contra esta bacteria sobre la base del PsC, constituyen formulaciones multivalentes (Didrik, 2010; Moutschen, 2016).

En Cuba, desde hace unos años, se trabaja en un proyecto de investigación para la obtención de una vacuna conjugada contra neumococos. La formulación contiene en la forma terminada siete IFAs de Ps de $S$. pneumoniae de los serotipos 1, 5, $6 \mathrm{~B}, 14,18 \mathrm{C}, 19 \mathrm{~F}$ y $23 \mathrm{~F}$ enlazados covalentemente al toxoide tetánico (TT) y adsorbidos en fosfato de aluminio como adyuvante.

Los métodos que permitan identificar o cuantificar los principios activos presentes en las formulaciones (el PsC en este caso) están dentro de los requisito obligatorio para la liberación final del producto (CEDMED, 2000; OMS, 2010). Estas organizaciones regulatorias recomiendan, para estos fármacos, que la prueba de identidad se realice por técnicas de RMN o inmunoquímicas como Western Blot y Dot Blot, utilizando an- ticuerpos monoclonales (AcM), pues además de su alta especificidad y sensibilidad, son métodos rápidos y de fácil realización (Ochoa, 2013; González 2015).

El objetivo del presente trabajo, fue establecer las condiciones analíticas que permitan utilizar la técnica del Dot.Blot para determinar la identidad del polisacárido capsular de S. pneumoniae serotipo $14 \mathrm{y}$ del toxoide tetánico presentes en vacunas conjugadas.

\section{MATERIALES Y MÉTODOS}

\section{Muestras}

Anticuerpos Monoclonales: Los AcM murinos utilizados fueron: 1D1D11 AcM anti PsC de S. pneumoniae serotipo 14 (PsC 14) y 10C7B12 AcM anti TT obtenidos en el laboratorio de AcM del Instituto Finlay de Vacunas.

Polisacárido de $S$. pneumoniae serotipo 14. Se utilizaron los lotes de: IFA 1202, 1302 y 1501; lotes del candidato vacunal conjugado heptavalente 1001, 1101, 1302 y 1303; se utilizaron muestras controles de IFA de los otros serotipos presentes en el candidato vacunal heptavalente $(1 ; 5 ; 6 \mathrm{~B}$; 18C; $19 \mathrm{~F}$ y 23F). Todas las muestras empleadas fueron obtenidas en el Instituto Finlay de vacunas en condiciones GMP.

Dot Blot. Para la realización del Dot Blot, se utilizó la metodología descrita previamente por González-Aznar (2010). Brevemente, se utilizó un equipo Minifold II S\&S (Alemania) acoplado a una bomba de vacío y se empleó una membrana de nitrocelulosa (MNC) de 0,45 $\mathrm{mm}$ (Bio-Rad, EUA) remojada previamente en Solución Salina Tamponada con Fosfato (SSTF), las muestras fueron aplicadas variando los volúmenes y concentraciones de las muestras y del AcM según el propósito de las determinaciones a realizar.

El Bloqueo de los sitios de uniones inespecíficas se realizó con leche descremada 3\% w/v en SSTF durante $30 \mathrm{~min}$ a $37^{\circ} \mathrm{C}$ utilizando un agitador orbitálico. A continuación se lavó 3 veces con $0,1 \%$ v/v de Tween 20 en SSTF (tampón de lavado) durante 5 minutos, para luego incubar la 
membrana con el AcM diluido en leche descremada $1 \% \mathrm{~m} / \mathrm{v}$; se incubó durante $30 \mathrm{~min}$ a $37^{\circ} \mathrm{C} \mathrm{C}$ con agitación lenta. Se lavó 3 veces durante $5 \mathrm{~min}$ con tampón de lavado y se incubó posteriormente con un conjugado anti IgG de ratón (Sigma) diluido $1 / 5000$ en leche descremada $1 \% \mathrm{~m} / \mathrm{v}$ durante $30 \mathrm{~min}$ a $37^{\circ} \mathrm{C}$ en agitación lenta. Posteriormente se lavó 3 veces con tampón de lavado durante $10 \mathrm{~min}$. Se reveló con un sistema de 3,3-tetrahidrocloro Di-amino benzidina (DAB) $-\mathrm{H}_{2} \mathrm{O}_{2}$ por $5 \mathrm{~min}$ y se detuvo la reacción de revelado con abundante agua, posteriormente se dejó secar la membrana y se guardó.

Determinación de los volúmenes de aplicación óptimos para las IFAs y vacunas: Para esta determinación se utilizó un lote de IFA de un conjugado monovalente del PsC de $S$. pneumoniae serotipo 19F (Lote: 1301) diluido $1 / 10(\mathrm{v} / \mathrm{v})$ y fueron ensayados volúmenes de $10,20,50$ y $100 \mu \mathrm{L}$ y del candidato vacunal conjugado heptavalente (Lote: Neu-1302) aplicada directamente del bulbo sin dilución previa. Estas condiciones y muestras fueron las mismas que se emplearon para el estudio con el AcM contra el TT.

Determinación de las concentraciones óptimas para las IFAs, vacunas y para los AcM: Se utilizaron concentraciones de 2, 5 y $10 \mu \mathrm{g} / \mathrm{mL}$ del AcM contra el PsC 14, como muestra antigénica se utilizó una IFA del PsC 14 conjugado a TT (Lote: 1501) en diluciones dobles seriadas a par-

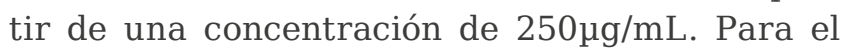
AcM anti TT las concentraciones utilizadas en el estudio fueron de 2.5, 5 y $10 \mu \mathrm{g} / \mathrm{mL}$ y se empleó el Lote: 1301 de la IFA del PsC 19F a una concentración de $125 \mu \mathrm{g} / \mathrm{mL}$. También se aplicó, en una tira y para cada valor de concentración del AcM en estudio, una muestra de un lote del candidato vacunal conjugado heptavalente (Lote: Neu1302).

Prueba de identidad del PsC 14 en lotes de IFAs y vacunas: Para la identidad del PsC en las IFAs de conjugados monovalentes de PsC 14 se utilizaron los lotes 1202, 1302 y 1501 a $125 \mu \mathrm{g} / \mathrm{ml}$ determinado por un ELISA. Además se analizaron cuatro lotes del candidato vacunal conjugado heptavalente (Lotes: 1001, 1101, 1302 y 1303) los cuales se aplicaron sin diluir. La concentración de trabajo y el volumen de aplicación para los AcM anti PsC 14 y anti TT fue el determinado en los acápites anteriores. También se utilizaron, como controles negativos para el PsC 14, dos IFAs de conjugados monovalentes del serotipo 19F (Lotes 19F-1202 y 19F-1501). Estas muestras fueron preparadas a la misma concentración que las IFAs de conjugados monovalentes del PsC 14. El volumen de aplicación para todas las muestras de estudio y controles fue de $10 \mu \mathrm{L}$.

Prueba de identidad del toxoide tetánico: La identidad del TT en las IFAs, de conjugados monovalentes, se realizó tomando muestras de lotes de los sietes conjugados monovalentes de $S$. pneumoniae (Sp 1 lote: 1301; Sp5 lote: 1301; Sp6B lote: 1401; Sp14 lote: 1501; Sp18C lote: 1501; Sp19F lote: 1501 y Sp23F lote: 1401) que componen el candidato vacunal heptavalente. Para el caso de las vacunas, los lotes del candidato vacunal heptavalente que se ensayaron fueron los lotes: 1101, 1302 y 1303. Además se utilizaron en este ensayo, muestras de toxoide diftérico (TD) y albumina de huevo (OVA; Merck) como controles negativos; todas preparadas a una concentración de $0.5 \mathrm{mg} /$ $\mathrm{ml}$. Las muestras empleadas, fueron obtenidas en el Instituto Finlay de vacuna en condiciones GMP. El volumen de aplicación para todas las muestras de estudio y controles fue de $10 \mu \mathrm{L}$.

Captura de las imágenes: Las imágenes de los Dot Blot fueron capturadas utilizando un densitómetro GS-800 (Bio-Rad, EUA) con software de captura y cuantificación (Quantity One).

\section{RESULTADOS Y DISCUSIÓN}

\section{Determinación del volumen de aplicación ópti- mo para las IFAs y vacunas.}

Para determinar el volumen de aplicación de las IFAs y vacunas en estudio se utilizó una IFA de un conjugado monovalente del PsC de S. pneumoniae serotipo $19 \mathrm{~F}$ que había sido evaluado en el laboratorio previamente. Los resultados se muestran en la figura 1.

Como se puede observar en la figura anterior, en todos los volúmenes aplicados se obtuvo un reconocimiento específico por los AcM a su antí-

\section{Cumbres}




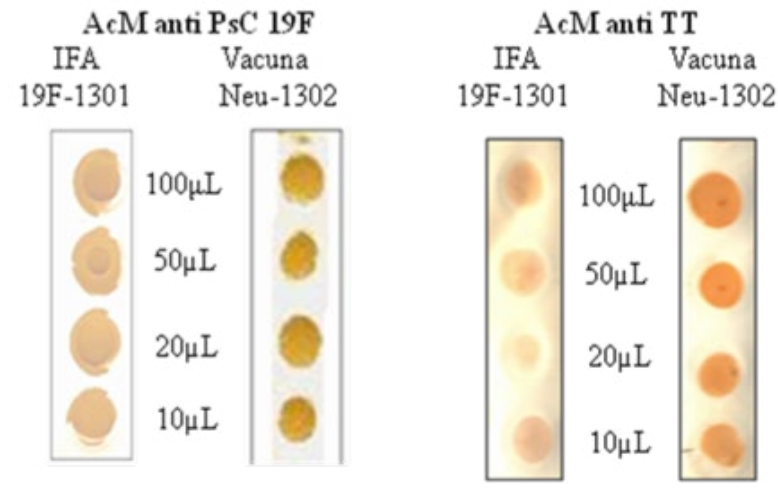

Fig. 1. Resultados del Dot Blot para determinar volumen de aplicación de las IFAs y formulaciones vacunales. IFA 19F-1301 a una dilución de 1/10 (vol/vol) y del lote Neu 1302 del candidato vacunal heptavalente conjugado contra neumococo sin previa dilución.

geno. Estos resultados demostraron que para la determinación de la identidad antigénica fueron suficientes $10 \mu \mathrm{L}$ de muestras de los conjugados monovalentes en una dilución de 1/10 (Vol/Vol) e igual volumen para las vacunas heptavalentes.

En el Dot Blot el volumen de las muestras a aplicar es un parámetro crítico, ya que un volumen grande de muestra pudiera difundir en la membrana hasta unirse con otra muestra que se haya aplicado en la misma tira de nitrocelulosa y dar como resultado un falso positivo y por el contrario, un volumen muy pequeño pudiera no contener la cantidad de antígeno necesaria para la visualización de la reacción antígeno-Ac. Los resultados obtenidos en este trabajo nos garantizan que con $10 \mu \mathrm{L}$ de estas muestras se obtienen las manchas que evidencian el reconocimiento antigénico por parte del AcM específico a dicho antígeno.

Determinación de las concentraciones óptimas para las IFAs, vacunas y para los AcM.

En la figura 2 se muestran los resultados de la determinación de la concentración óptima de trabajo para las IFAs y de los AcM anti PsC 14 y anti TT.

Como puede observarse se obtuvieron manchas para todas las concentraciones de AcM estudiadas, tanto para el AcM anti PsC 14 como para el AcM anti TT.

En el estudio de las concentraciones de las IFAs y vacunas podemos observar que para el

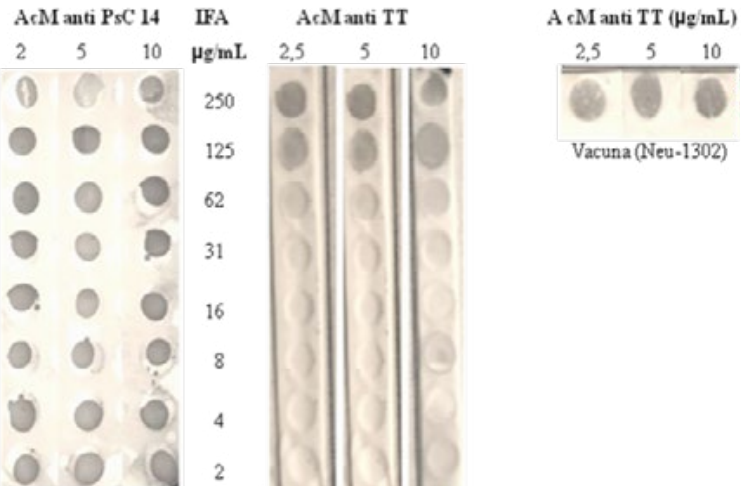

Fig. 2. Resultados del estudio de las concentraciones de los AcM anti PsC 14 y anti TT, muestras de una IFA del PsC 14 conjugado a TT (Lote: 1501) y de la vacuna heptavalente contra el neumococo (Lote: Neu1302). Se estudiaron las concentraciones de 2, 5 y $10 \mu \mathrm{g} / \mathrm{ml}$ del AcM anti PsC 14 y 2.5, 5 y $10 \mu \mathrm{g} / \mathrm{ml}$ del AcM anti TT y diluciones dobles seriadas de la IFA.

AcM anti PsC 14 se obtuvieron manchas para todas las concentraciones estudiadas, mientras que para el AcM anti TT la menor concentración a la que se pudo observar una mancha fue a $62 \mu \mathrm{g} / \mathrm{mL}$ en la IFA del PsC 14, siendo esta mancha débil a cualquiera de las tres concentraciones de AcM anti TT empleadas. A la menor concentración del AcM anti TT $(2,5 \mu \mathrm{g} / \mathrm{mL})$ que se obtuvo una mancha fuerte fue a $125 \mathrm{\mu g} / \mathrm{mL}$ de la IFA; mientras que en la vacuna se obtuvo una mancha oscura a las 3 concentraciones del AcM estudiadas, por lo que se decidió continuar el estudio en las condiciones de $125 \mu \mathrm{g} / \mathrm{mL}$ de IFA y la vacuna directamente del bulbo con 2 y 2,5 $\mu \mathrm{g} / \mathrm{mL}$ de AcM anti PsC 14 y TT respectivamente.

En las técnicas inmunoenzimáticas como el Dot Blot, Western Blot o ELISAs, hay una etapa de inmovilización de la muestra sobre el soporte analítico (MNC o placa de 96 pocillos, según la técnica a utilizar) y otra etapa de reacción del antígeno con el anticuerpo, en estas dos etapas es muy importante determinar la concentración óptima tanto del antígeno como del Ac que se emplee, ya que la relación entre estas dos deben estar en el rango de la zona de equivalencia para que ocurra la formación de los complejos Ag-Ac en una cantidad que pueda ser visualizada posteriormente con el empleo de la reacción con la solución reveladora (Ochoa, 2013), que en nuestro caso utilizamos la 3,3-tetrahidrocloro Di-amino benzidina (DAB) al 0,04\% y $\mathrm{H}_{2} \mathrm{O}_{2}$ al 0,5\%. 
Está reportado que concentraciones no óptimas del anticuerpo pueden influir negativamente en la sensibilidad y en el límite de detección del ensayo, ocasionando lo que se conoce como efecto gancho. También está reportado que concentraciones entre $1-10 \mu \mathrm{g} / \mathrm{mL}$ del Ac son suficientes (Ochoa, 2013) para visualizar un reconocimiento entre el antígeno y los anticuerpos en estos ensayos inmunnoenzimáticos; lo cual concuerda con los resultados obtenidos en este trabajo, donde con $125 \mu \mathrm{g} / \mathrm{mL}$ de antígeno y 2 y $2,5 \mu \mathrm{g} / \mathrm{mL}$ de AcM anti PsC 14 y anti TT respectivamente, obtuvimos manchas de reconocimiento evidente.

\section{Prueba de identidad del PsC 14 en lotes de IFAs y vacunas.}

Una vez establecidos los parámetros de trabajo para las muestras a evaluar y el AcM anti PsC 14, pasamos a evaluar su comportamiento con otras muestras de IFAs y vacunas. Los resultados se muestran en la figura 3.

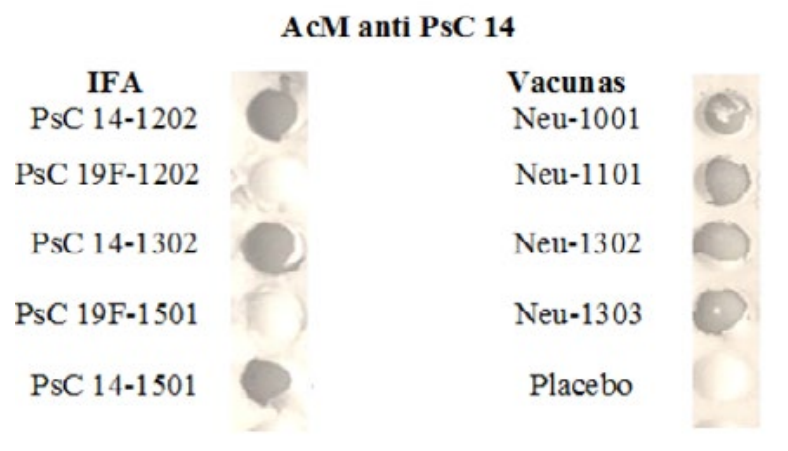

Fig. 3. Resultados del estudio de identidad de 3 lotes de IFAS contra PsC 14, 2 PsC 19F conjugado a TT y de 4 lotes de la vacuna heptavalente contra el neumococo; placebo, fosfato de aluminio, adyuvante empleado en las vacunas.

En la figura 3 se muestra que los AcM fueron capaces de identificar en cada lote de IFA evaluado, su correspondiente PsC de forma específica, no evidenciándose señal en los pozos de lotes que no tenían en su formulación el PsC 14, lo mismo ocurrió con los lotes de vacunas aplicada donde se observó la mancha característica de la reacción antígeno anticuerpo, producto del reconocimiento especifico en todos los lotes estudiados y discriminando a la muestra placebo utilizada como control negativo. Este resultado es una evidencia de la especificidad del AcM anti PsC 14 que solo reconoce a este PsC.

\section{Prueba de identidad del toxoide tetánico}

Para el AcM anti PsC TT, pasamos a evaluar su comportamiento con muestras de IFAs de los conjugados monovalentes que están presentes en el candidato vacunal heptavalente; en todos estos conjugados está presente el TT como proteína transportadora; también fueron evaluadas tres lotes del candidato vacunal heptavalente. Los resultados son mostrados en la figura 4 .

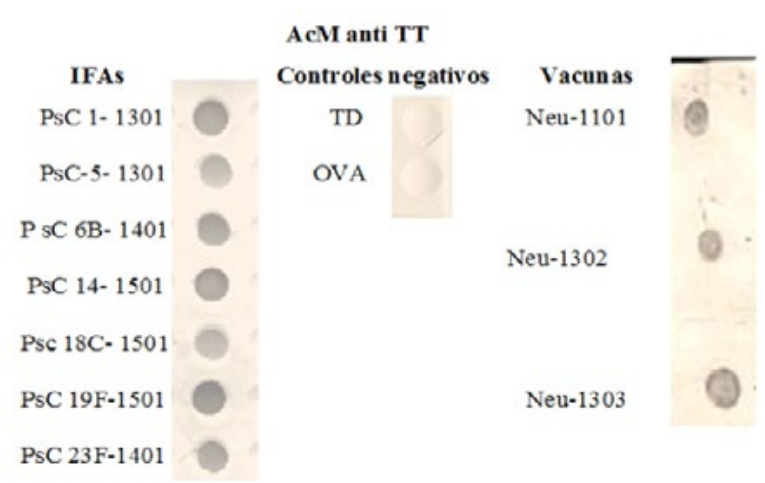

Fig. 4. Identidad antigénica del TT en IFAs de conjugados monovalentes de los diferentes serotipos que componen al candidato vacunal heptavalente conjugados todos al TT. También se utilizaron tres lotes del candidato vacunal heptavalente conjugado (Neu: 1101, 1302 y 1303). Se utilizó OVA y DT como controles negativos.

En los resultados anteriores se observa que hubo reconocimiento específico del AcM al TT en todas las muestras que tenían el TT como proteína portadora en la conjugación, y no hubo reconocimiento ni contra el DT ni la Ova, proteínas utilizadas en este estudio como control negativo; lo cual es una evidencia de la especificidad de este AcM anti TT ya que no reconoció a otra proteína que no sea el TT.

Uno de los requerimientos de los organismos regulatorios para la liberación de los lotes de vacunas para ser utilizadas en humanos, es la determinación de la identidad antigénica de los componentes vacunales, la cual puede determinarse por métodos serológicos con el uso de Ac 
específicos a los PsC y proteínas que componen dichas vacunas (WHO, 2009). Los resultados obtenidos en este trabajo, tanto para determinar la identidad del PsC 14 como para el TT, son una evidencia de que contamos con una metodología que daría cumplimiento a este requerimiento de los organismos regulatorios, siempre que se utilice en las condiciones de trabajo aquí expuestas.

Como conclusiones podemos plantear que quedaron establecidas las condiciones de trabajo en el laboratorio para determinar la identidad del polisacárido capsular de $S$. pneumoniae serotipo 14 y para el toxoide tetánico en las vacunas conjugadas multivalentes por el método del Dot Blot.

\section{REFERENCIAS BIBLIOGRÁFICAS}

Cabrera, O.; Cuello, M.; Soto, C.; Martínez, M.; Del Campo, J.; Pérez, O.; Infante, J. \& Sierra, G. (2007). New Method For Obtaining Conjugated Vaccines Vaccine 24: S76-S78

CECMED. (2000). Requisitos para la liberación de lotes de vacunas. Regulación No.19-2000. La Habana: CECMED.

Vestrheim, D.; Hoiby, E.; Aaberge, I. \& Caugant, D. (2010). Impact of a Pneumococcal Conjugate Vaccination Program on Carriage among Children in Norway. Clin Vaccine Immunol. 17(3): 325-334.

González-Aznar, E., Otero-Alfaro, O., Cabrera-Blanco, O., Ramírez-Bencomo, F., Fajardo-Sánchez, A., Mandariote-Llanes, A. \& Cuello-Pérez, M. (2015). Evaluación de los Anticuerpos Monoclonales anti-polisacárido capsular de Neisseria meningitidis serogrupos A, C, Y, W y X para su uso en los ensayos de identidad. Vaccimonitor, 24(2), 64-70.

Ministerio de Salud Pública (2015). Anuario Estadístico de Salud. Recuperado de http://www.sld. cu/sitios/dne

Moutschen M. (2016). Prevenar 13®, A Pneumococcal Conjugate Vaccine For The Prevention of Pneumococcal Infections In Adults At Risk. Rev. Med. Liege. 71(4):204-9.

Ochoa, R. F. (2013) Técnicas inmunoenzimáticas en el desarrollo clínico de vacunas. La Habana: Finlay
Ediciones.

OMS (2015). Neumonía. Nota Descriptiva No. 331. Recuperado de: http://www.who.int/mediacentre/factsheets/fs331/es/

Ortqvist, A.; Hedlund, J.; Kalin, M. (2005). Streptococcus pneumoniae: epidemiology, risk factors, and clinical features. Semin Respir Crit Care Med. 26(6):563-74.

World Health Organization (2009). Expert Committee on Biological Standardization. Geneva, 19 to 23 October 2009. Recommendations to assure the quality, safety and efficacy of pneumococcal conjugate vaccines. Replacement of: TRS 927, Annex 2. 